\title{
Mechanism of apoptosis involved in gastric mucosal lesions in Tibetans with high-altitude polycythemia
}

\author{
$\mathrm{KANG} \mathrm{LI}^{1,2}$, LUOBU GESANG ${ }^{2}$ and CHAOHUI HE ${ }^{1}$ \\ ${ }^{1}$ Department of Gastroenterology, The Fifth Affiliated Hospital of Zunyi Medical University, Zhuhai, \\ Guangdong 519100; ${ }^{2}$ Department of Gastroenterology, People's Hospital of Tibet Autonomous Region, \\ Lhasa, Tibet 850000 , P.R. China
}

Received July 14, 2016; Accepted April 7, 2017

DOI: $10.3892 / \mathrm{etm} .2017 .4996$

\begin{abstract}
The Tibetan high plateau is a low-oxygen environment, which may cause the pathogenesis of high-altitude polycythemia (HAPC). Gastric mucosal lesions (GML) are a common complication of HAPC. The molecular mechanisms involved in HAPC-induced GML have remained to be fully elucidated and were therefore investigated in the present study. Gastric tissues of patients with heavy, HAPC-induced GML and healthy controls were assessed by ultrastructural and histopathological analysis. In addition, terminal deoxynucleotidyl transferase-mediated deoxyuridine triphosphate nick-end labeling and reverse transcription-quantitative polymerase chain reaction (RT-qPCR) analysis were used to detect cell apoptosis in the gastric mucosa tissues. Moreover, the expression of genes associated with the phosphoinositide-3 kinase (PI3K) pathway was assessed by RT-qPCR to investigate the mechanism of cell apoptosis in HAPC-induced GML. The results revealed a significant increase in the number of red blood cells, gastric vessels and the diameter of gastric mucosal vessels in HAPC-induced GML patients compared with those in healthy controls. In addition, more red blood cells were distributed in gastric tissue not only at the vascular level but also in the tissue space. The number of vacuoles was increased in the gastric mucosal cells. Furthermore, a
\end{abstract}

Correspondence to: Dr Kang Li, Department of Gastroenterology, The Fifth Affiliated Hospital of Zunyi Medical University, 1439 Mount Everest Avenue, Doumen, Zhuhai, Guangdong 519100, P.R. China

E-mail: likang820@aliyun.com

Abbreviations: HAPC, high-altitude polycythemia; GML, gastric mucosal lesion; RBC, red blood cell; Hb, hemoglobin; RT-qPCR, Reverse transcription-quantitative polymerase chain reaction; TUNEL, terminal deoxynucleotidyl transferase-mediated deoxyuridine triphosphate nick-end labeling; BSA, bovine serum albumin; HRP, horseradish peroxidase; PI3K, phosphatidylinositide-3 kinase; mTOR, mammalian target of rapamycin

Key words: high-altitude polycythemia, gastric mucosal lesion, apoptosis, PTEN, mechanism significant increase in apoptosis of the gastric mucosal cells was identified. The expression of phosphatase and tensin homolog was significantly higher in gastric mucosa from patients with HAPC-induced GML compared with that in the healthy controls. All of the pathologic changes suggested that significant cell apoptosis occurred in the HAPC-induced GML tissues, which may be associated with the PI3K pathway. These findings may provide novel insight for the treatment of gastric lesions caused by HAPC in the future.

\section{Introduction}

At present, almost $18 \%$ of the population residing in the Tibetan Plateau suffer from high-altitude polycythemia (HAPC), which is characterized by excessive erythrocytosis [females, hemoglobin $(\mathrm{Hb})>19 \mathrm{~g} / \mathrm{dl}$; males, $\mathrm{Hb}>21 \mathrm{~g} / \mathrm{dl}$ ] (1). At high altitudes, hypoxia is usually encountered and may cause various symptoms, such as anemia, which are associated with the deficiency of red blood cells (RBCs) and/or hemoglobin and reduce the ability of the body to transfer oxygen to tissues (2). During long-term exposure to high altitudes, the RBC count increases in order to provide sufficient oxygen supply (3). While the RBC count stabilizes at a certain level in most individuals, continuous increases of RBCs may give rise to serious pathologies.

A previous study reported that digestive symptoms such as epigastric pain, dyspepsia, anorexia, vomiting and diarrhea, which frequently occur in patients with HAPC, may be eliminated in $89-100 \%$ of cases (4). Gastric mucosal lesions (GML) are a common complication of HAPC. It has been demonstrated that blood flowing in the micro-vessels (capillaries, arterioles and collecting venues) has an important role in the maintenance of the structure and function of all tissues, including the gastrointestinal mucosa $(5,6)$. Under certain circumstances, the gastric microcirculation is bypassed by arteriovenous shunting, resulting in severe gastric mucosal injury (7). Therefore, gastric mucosal ischemia caused by microvascular thrombosis due to excessive polycythemia may contribute to the HAPC-induced GML.

In addition, long-term high-altitude hypoxia has varying effects on the digestive system. The gastrointestinal tract has increased sensitivity to hypoxia and is one of the systems commonly affected by it (8). Syam et al (9) demonstrated 
that hypoxia leads to more severe gastric ulceration using a systemic hypoxia rat model. Though we have found some genes differentially expressed in HAPC-induced GML, the understanding of the underlying molecular mechanisms is currently limited (10).

Therefore, the present study investigated the role of a high-altitude hypoxic environment in the physiological function of human gastrointestinal mucosa and explored the molecular mechanisms involved in the pathogenesis of HAPC-induced GML in a Tibetan population.

\section{Materials and methods}

Subjects. The experimental protocol was established according to the ethical guidelines of the Helsinki Declaration and approved by the ethics committee of People's Hospital of Tibet Autonomous Region (Lhasa, China). Written informed consent was obtained from the individual participants.

The severity of GML was classified according to hematoxylin and eosin (H\&E) staining of gastric tissues and RBC concentrations: i) Healthy controls with healthy stomachs, without RBC aggregation or leakage, and/or seepage in gastric tissues; ii) HAPC patients, RBCs ooze and cluster together (>3 erythrocytes were regarded as a cluster). The categories were determined according to the number of RBCs and vessels, the aggregation degree of RBCs and pathological images. In July 2013, 11 HAPC patients residing on the Tibetan Plateau at an average altitude of $3600-4800 \mathrm{~m}$, with a definite diagnosis of HAPC, were enrolled in a HAPC group, and all had symptoms that justified gastroscopy. In addition, 13 healthy Tibetan individuals residing in similar high altitudes, who had screening requirements for gastrointestinal endoscopic examination during the corresponding period, were enrolled as a control group. All subjects were from Lhasa, Nagqu, Shannan or Rigaze of Tibet (China) and all of them were indigenous and had lived in their regions for at least 30 years. Each patient was matched to a control according to sex, birthplace, age, lifestyle, diet, body mass index (BMI), height of location and work intensity. As BMI and per capita income are main factors affecting gastric injury caused by inflammation (11), these factors were carefully considered. All of the subjects were native male Tibetans from Lhasa aged 40-45 years. All the subjects were asked to sign an informed consent form prior to tissue biopsy.

Prior to endoscopy, peripheral venous blood was sampled for a routine blood test. Pulse oximetry was used to measure the oxygen saturation of arterial blood. Inclusion criteria for the study were as follows: HAPC defined by the 2004 Qinghai International High Altitude Medicine Conference as a $\mathrm{Hb}$ concentration of $>21 \mathrm{~g} / \mathrm{dl}$ (males) and $>19 \mathrm{~g} / \mathrm{dl}$ (females) (1).

Exclusion criteria were as follows: Chronic pulmonary diseases, including emphysema, bronchitis, bronchiectasis, alveolar fibrosis, lung cancer and other serious pulmonary diseases; chronic respiratory disorders or secondary polycythemia due to hypoxemia caused by certain chronic diseases; severe diseases of the heart, brain, lungs, liver, kidneys, endocrine system and hematopoietic system; alcohol abuse, drug addiction, poor mental health or other conditions rendering patients unsuitable for gastroscopy; obstructed gastrointestinal tract and medical histories such as recent gastrointestinal bleeding. The diagnosis of chronic gastritis was made according to the Chinese Consensus on Chronic Gastritis from 2006 (12). The histopathological diagnosis was based on the Operative Link on Gastritis Assessment staging system (12).

Endoscopic examination. Rigorous endoscopic surveillance was maintained for all subjects (13). HAPC was measured after oral administration of $10 \mathrm{ml}$ of viscous lidocaine hydrochloride Mucilage (Jiangsu Jichuan Pharmaceutical Co., Ltd., Huaian, China) in all subjects. A gastroscope (GIF-260; Olympus, Tokyo, Japan) was used for examination. All subjects were examined using the same model of endoscope by an experienced endoscopist. The light source strength and types of endoscopic lamps were consistent throughout the study. The color of esophageal, gastric and duodenal mucosal lesions was carefully assessed. The color changes of the upper gastrointestinal mucosa for patients with HAPC were determined by direct endoscopic observation. Images of the upper gastrointestinal tract were captured, including the esophagus, cardia, gastric fundus, gastric antrum and body, the duodenal bulb and the descending portion. Endoscopic mucosal biopsy specimens were obtained in all subjects.

Histological analysis. The mucosa was collected at the Digestive Endoscopy Department of People's Hospital of the Tibet Autonomous Region (Lhasa, China). In total, 24 gastric antrum biopsies were analyzed, comprising 11 samples from HAPC patients and 13 from controls with normal underlining mucosa. The samples were snap-frozen in liquid nitrogen immediately after obtaining forceps biopsies of gastric mucosa tissue and stored at $-80^{\circ} \mathrm{C}$ until use. Gastric mucosal tissues were fixed in 4\% paraformaldehyde solution (cat. no. AR1068; Wuhan Boster Biological Technology, Ltd., Wuhan, China) and embedded in paraffin for histological analyses. Sections (5 $\mu \mathrm{m}$ ) were stained with hematoxylin and eosin (Bi Yuntian, Wuhan, China). Under a microscope with a magnification of $\mathrm{x} 400$, blood vessels and RBCs inside micro-vessels were counted manually, and the diameter of five randomly selected vessels was measured. The mucosa was considered injured if one or more of the following criteria were met: Discontinuous surface, dilated gland, hemorrhage or damage of superficial cells.

Immunohistochemical analysis. Paraffin sections of Gastric mucosa tissue at $5 \mu \mathrm{m}$ thick were incubated in $0.3 \%$ hydrogen peroxide methanol solution for $15 \mathrm{~min}$. A total of $100 \mu \mathrm{l}$ normal fetal bovine serum (Gibco; Thermo Fisher Scientific, Inc., Waltham, MA, USA) was added, followed by further culture for $10 \mathrm{~min}$ at room temperature to block the sections. Subsequently, anti-PTEN antibody (rabbit anti-human; 1:200 dilution; cat. no. ab31392; Abcam, Cambridge, MA, USA) was added, followed by incubation at $4^{\circ} \mathrm{C}$ for $16 \mathrm{~h}$. Subsequent to three washes with PBS, horseradish peroxidase (HRP)-conjugated goat anti-rabbit secondary antibody (goat anti-rabbit; 1:200 dilution; cat. no. sp-9001, Zhongshang Goldenbridge Bio, Beijing, China) was added, followed by incubation for $30 \mathrm{~min}$. Finally, after three washes, samples were developed with a diaminobenzidine color kit (Zhongshang Goldenbridge Bio, 
Beijing, China). The samples were stained with hematoxylin and then mounted. The expression and distribution of PTEN was observed in the tissue.

Western blot analysis. Protein was extracted from Glandular epithelial cells isolated from gastric mucosa and PTEN was assayed by western blotting. Cells were collected with a proteinase inhibitor cocktail ( $2 \mathrm{mM}$ N-ethylmaleimide, $2 \mathrm{mg} / \mathrm{ml}$ aprotinin, $4 \mathrm{mg} / \mathrm{ml}$ pepstatin, $10 \mathrm{mg} / \mathrm{ml}$ leupeptin and $2 \mathrm{mM}$ phenylmethylsulphonyl fluoride; Thermo Fisher Scientific, Inc.) and lysed by three-cycle freezing and thawing. Then protein concentration was determined using the BCA Protein Assay kit (cat. no. AR0146; Wuhan Boster Biological Technology, Ltd.). The supernatant samples were solubilized in SDS-PAGE buffer containing $50 \mathrm{mM}$ Tris $\mathrm{HCl}, 10 \%$ glycerol, $1 \%$ SDS, $0.05 \%$ bromophenol blue and $5 \% \beta$-mercaptoethanol and the $\mathrm{pH}$ was adjusted to 6.8. A total of $10 \mu \mathrm{g}$ protein from each cell lysate was separated by $10 \%$ SDS-PAGE at $200 \mathrm{~V}$ for $2 \mathrm{~h}$. The positive antigen controls were $0.5 \mu \mathrm{g}$ PTEN (Sigma-Aldrich; Merck KGaA, Darmstadt, Germany). After electrophoresis, the separated proteins were transferred to a nitrocellulose membrane (cat. no. 66485; Pall Corporations, New York, NY, USA) in transfer buffer $(48 \mathrm{mmol} / 1 \mathrm{Tris}, 39 \mathrm{mmol} / \mathrm{l}$ glycine, $20 \%$ methanol, $\mathrm{pH}$ 9.2) for $2 \mathrm{~h}$ at a constant current of $20 \mathrm{amps}$ and blocked in Tris-buffered saline containing $2.5 \%$ non-fat dry milk for $30 \mathrm{~min}$. The membranes were incubated overnight at $4^{\circ} \mathrm{C}$. Secondary antibodies were then added against PTEN, including Rabbit anti-human primary antibody (1:200; cat. no. ab31392; Abcam) and Goat anti-rabbit secondary antibody, Protein A-HRP (cat. no. BA1080; 1:2,000; Wuhan Boster Biological Technology, Ltd.). $\beta$-actin (cat. no. AA128; 1:1,000, Beyotime Institute of Biotechnology) was used as the internal control. An avidin-biotin complex peroxidase kit and a peroxidase substrate kit (cat. nos. A0303 and P0202; Beyotime Institute of Biotechnology, Shanghai, China) were used to detect the primary antibodies bound to the antigen. Membranes were exposed to X-ray film, images were captured and the expression of PTEN was measured using Quantity One software (Bio-Rad Laboratories, Inc., Hercules, CA, USA).

Reverse transcription-quantitative polymerase chain reaction $(R T-q P C R)$. Total RNA was isolated from each gastric mucosal sample respectively using TRIzol reagent (Invitrogen; Thermo Fisher Scientific, Inc., Waltham, MA, USA) according to the manufacturer's instructions. After DNase (cat. no. RT411; Tiangen Biotech, Co., Ltd., Beijing, China) digestion, RNA was quantified and purity was assessed at 260/280 nm using an Agilent Bioanalyzer (Agilent Technologies, Palo Alto, CA, USA). Five micrograms of RNA were reverse-transcribed using a RT reaction kit (Applied Biosystems; Thermo Fisher Scientific, Inc.). Platinum TaqDNA polymerase was from Applied Biosystems (Thermo Fisher Scientific, Inc.). The primers were designed as follows: Phosphatase and tensin homolog (PTEN) forward, 5'-GATTCGACTTAGACTTGA CCT-3' and reverse, 5'-TTTGGCGGTGTCATAATGTCT-3'; phosphoinositide-3 kinase (PI3K) forward, 5'-CCTCAG GCTTGAAGAGTGTCG-3' and reverse, 5'-TTGCCGTAA ATCATCCCCAT-3'; AKT forward, 5'-TACTTCCTCCTC
AAGAATGATGGC-3' and reverse, 5'-GCAGCGGATGAT GAAGGTGT-3'; B-cell lymphoma 2-associated X protein (BAX) forward, 5'-TTTCTGACGGCAACTTCAACTG-3' and reverse, 5'-AGCACTCCCGCCACAAAGA-3'; caspase-3 forward, 5'-AGAACTGGACTGTGGCATTGAGA-3' and reverse, 5'-GCACAAAGCGACTGGATGAAC-3'; caspase-7 forward, 5'-GACTTCCTCTTCGCCTATTCCA-3' and reverse, 5'-GGCAACTCTGTCATTCACCCTG-3'; $\beta$-actin forward, 5'-GAAGATCAAGATCATTGCTCCT-3' and reverse, 5'-TAC TCCTGCTTGCTGATCCA-3'; probe, 5'-FAM-CTGTCCACC TTCCAGCAGA-TAMRA-3'. PCR was performed in triplicate using SYBR Green PCR Master Mix (Roche Holding AG, Basel, Switzerland) using a 7500 Fast real-time PCR detection system (Applied Biosystems; Thermo Fisher Scientific, Inc.) with the following amplification conditions: $94^{\circ} \mathrm{C}$ for $2 \mathrm{~min}$, 45 cycles of $94^{\circ} \mathrm{C}$ for $20 \mathrm{sec}, 53^{\circ} \mathrm{C}$ for $30 \mathrm{sec}$ and $60^{\circ} \mathrm{C}$ for $45 \mathrm{sec}$. The relative expression value was calculated via the $2^{-\Delta \Delta \mathrm{Cq}}$ method (14).

Terminal deoxynucleotidyl transferase (TDT)-mediated deoxyuridine triphosphate (dUTP) nick-end labeling (TUNEL) analysis. Nuclei of tissue sections that had not been used in the previous assays were stripped from proteins by incubation with $10 \mu \mathrm{g} / \mathrm{ml}$ proteinase K (Sigma-Aldrich; Merck KGaA) for 15 min at room temperature, followed by washing for $2 \mathrm{~min}$ in distilled water. Endogenous peroxidase was inactivated by covering the sections with $2 \% \mathrm{H}_{2} \mathrm{O}_{2}$ for $5 \mathrm{~min}$ at room temperature. The sections were rinsed with distilled water and immersed in TDT buffer $[25 \mathrm{mM}$ Tris- $\mathrm{HCl}$, pH 6.5, $200 \mathrm{mM}$ potassium cacodylate, $1 \mathrm{mM}$ cobalt chloride, $0.25 \mathrm{mg} / \mathrm{ml}$ bovine serum albumin (BSA, Sangon Biotech, Co., Ltd., Shanghai, China)]. To determine apoptosis of gastric mucosa by TUNEL assay, TDT $(200 \mathrm{U} / \mathrm{ml}$; Takara, Tokyo, Japan) and biotinylated dUTP $(0.01 \mathrm{mM}$; Bristol Myers Squibb, New York, NY, USA) in TDT buffer were then added to cover the sections, followed by incubation in a humidified atmosphere at $37^{\circ} \mathrm{C}$ for $60 \mathrm{~min}$. The reaction was terminated by transferring the slides to SSC $(300 \mathrm{mM}$ sodium chloride and $30 \mathrm{mM}$ sodium citrate) for $15 \mathrm{~min}$ at room temperature. The sections were rinsed with distilled water, covered with a $2 \%$ aqueous solution of BSA for $10 \mathrm{~min}$ at room temperature, rinsed in distilled water and immersed in PBS for $5 \mathrm{~min}$. The sections were covered with a Streptavidin-HRP solution (cat. no. A0303, Beyotime, Institute of Biotechnology) included in a streptavidin HRP detection system (Research Genetics, Huntsville, AL, USA) for $45 \mathrm{~min}$. The slides were then washed with $0.02 \mathrm{M}$ PBS containing $0.15 \mathrm{M} \mathrm{NaCl}$ and incubated with $0.02 \%$ diaminobenzidine colorimetric solution. After rinsing in distilled water, the slides were stained with Mayer's hematoxylin solution for $30 \mathrm{~min}$ at $37^{\circ} \mathrm{C}$. Dehydration was performed once in $95 \%$ ethanol, twice in $100 \%$ ethanol and twice in xylene. The tissue sections were then mounted in Permount (Thermo Fisher Scientific, Inc.). Apoptotic cells with positive TUNEL staining were quantitated under a bright-field microscope by a single observer. The cell number was counted in six high-power fields from each specimen.

Statistical analysis. Statistical analysis was performed using the SPSS 17.0 software package (SPSS Inc., Chicago, IL, USA). Values are expressed as the mean \pm standard deviation from 


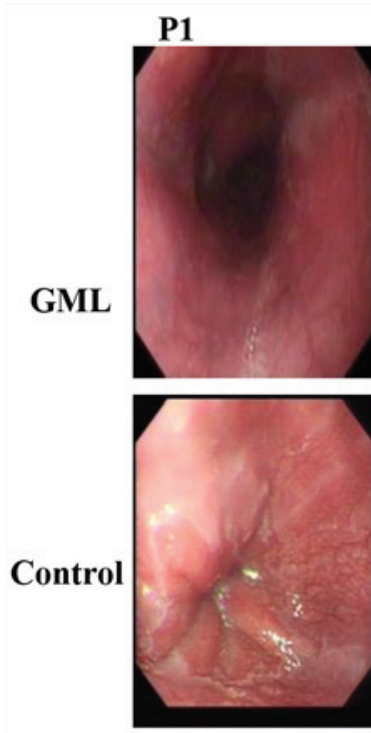

N1
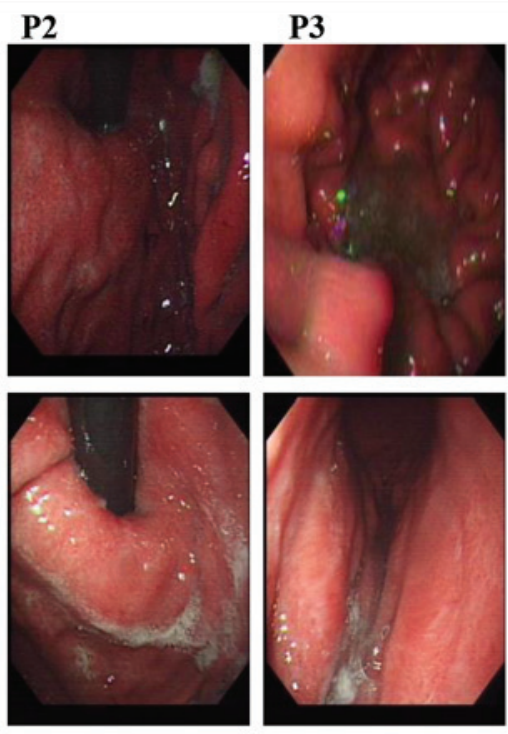

N2

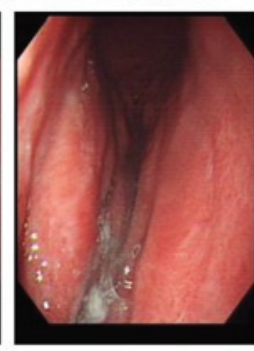

N3

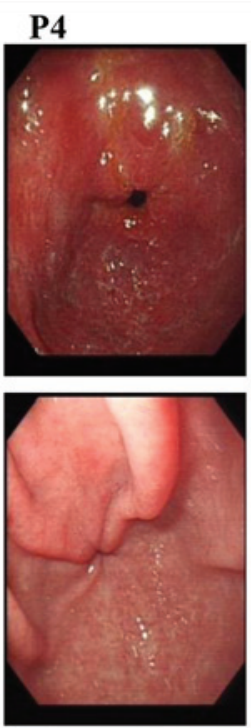

N4
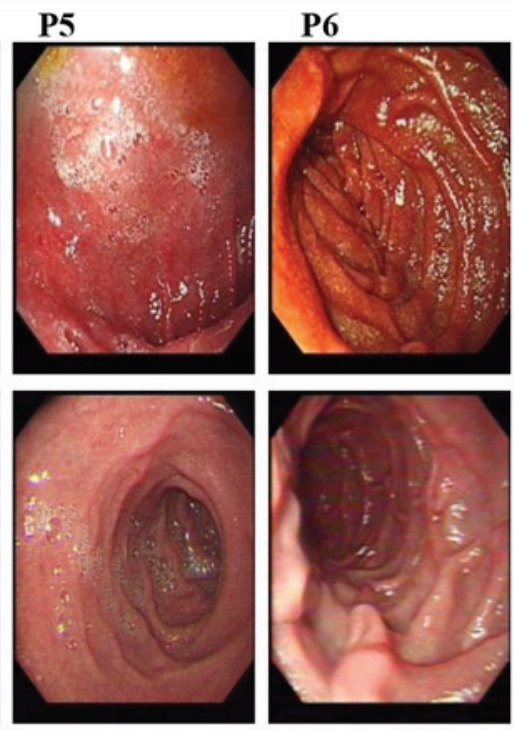

N5

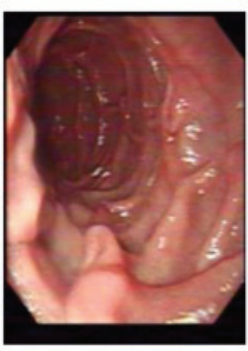

N6

Figure 1. Endoscopic examination of the upper gastrointestinal tract in patients with high-altitude polycythemia-induced gastric mucosal lesions and healthy controls. The upper gastrointestinal tract includes esophagus (P1/N1), cardia (P2/N2), gastric fundus (P3/N3), gastric antrum and body (P4/N4), the duodenal bulb (P5/N5) and descending portion (P6/N6). N, normal healthy subjects; P, polycythemia patients.

three independent experiments. Statistical differences between the groups were compared using Student's t-test. $\mathrm{P}<0.05$ was considered to indicate a statistically significant difference.

\section{Results}

Baseline characteristics of participants. In total, 11 HAPC patients and 13 healthy controls from the Tibetan high plateau were included in the present study. The characteristics of the two groups were compared. There was no significant difference in the distribution of living altitude between HAPC patients and healthy controls ( $\mathrm{P}>0.05)$; there was also no difference in other parameters, including age, cigarette smoking, alcohol consumption, BMI or years spent on different plateaus $(\mathrm{P}>0.05)$. The $\mathrm{Hb}$, erythropoietin, reactive oxygen species and diastolic pressure in the HAPC group were significantly higher than those in the control group $(\mathrm{P}<0.01)$. Notably, the oxygen saturation in the HAPC group was significantly lower than that in the control group $(\mathrm{P}<0.01)$.

Endoscopic examination of the upper gastrointestinal tract. In the HAPC group with evident mucosal hyperemia and edema, as presented in Fig. 1, endoscopic findings commonly showed a significantly darker color in the upper gastrointestinal tract, which includes esophagus (P1/N1), gastric fundus $(\mathrm{P} 2 / \mathrm{N} 2)$, gastric body $(\mathrm{P} 3 / \mathrm{N} 3)$, gastric antrum $(\mathrm{P} 4 / \mathrm{N} 4)$, the duodenal bulb (P5/N5) and descending portion (P6/N6). Furthermore, in the HAPC-induced GML group, the esophageal mucosa was thin and red and a fine meshwork of vessels was observed under the mucosa as cord- and branch-like structures. In addition, in GML patients, congestion was detected in areas where veins were slightly wider compared with those in the healthy controls. Furthermore, due to the significantly darker pink appearance of the esophageal mucosa, there were no evident boundaries between esophageal and gastric mucosa.
In the HAPC-induced GML group, the color of the gastric mucosa was deeper and darker, mainly manifesting as a dark red or red-purple color. No sign of diffuse hyperemia and edema or obvious changes of congestion were observed. Furthermore, in the duodenal bulb and descending segment of the in HAPC-induced GML group, the mucosal color had a higher brown component and resembled a brownish red compared to the control group. In addition, as shown in Fig. 1, the villi of the duodenal bulb were slightly enlarged with marked hyperemia and swelling.

Histopathological changes. Histopathological analysis revealed that in the healthy controls, the duct had a normal structure with suitable amounts of blood vessels and RBCs (Fig. 2A). By contrast, in the HAPC-induced GML patients, the duct was destroyed. A large number of RBCs were gathered in the gastric vessels. Parietal cells were over-proliferated in gastric biopsies. The number of gastric cells was decreased, while the volume and diameter of gastric vessels was increased compared with those in the healthy controls (Fig. 2A).

As shown in Fig. 2B, the number of gastric vascular vessels was significantly higher in the stomach tissues of HAPC-induced GML patients than that in the healthy controls $(23.88 \pm 4.46$

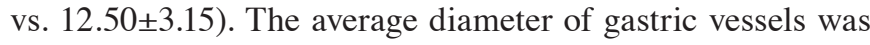
significantly higher in the stomach tissue of HAPC-induced GML patients than that in the healthy controls $(30.84 \pm 10.52$ vs. 13.33 \pm 4.19 ; Fig. 2C) In addition, the number of erythrocytes in gastric mucosa was significantly higher in the stomach tissue of HAPC-induced GML patients than that in healthy controls (150.92 \pm 67.84 vs. $29.71 \pm 14.18$; Fig. $2 D)$, which showed that erythrocytes are distributed in blood vessels together with tissue space, suggesting that high altitude may lead to the increase the number of gastric vessels and cause injury of the stomach.

Cell apoptosis in HAPC-induced GML. To explore the cell biological mechanisms of HAPC-induced GML, TUNEL 
A

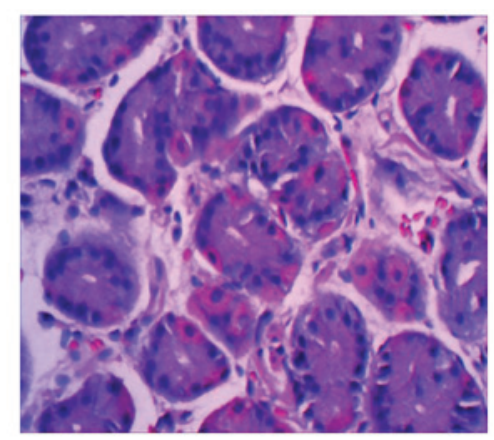

B

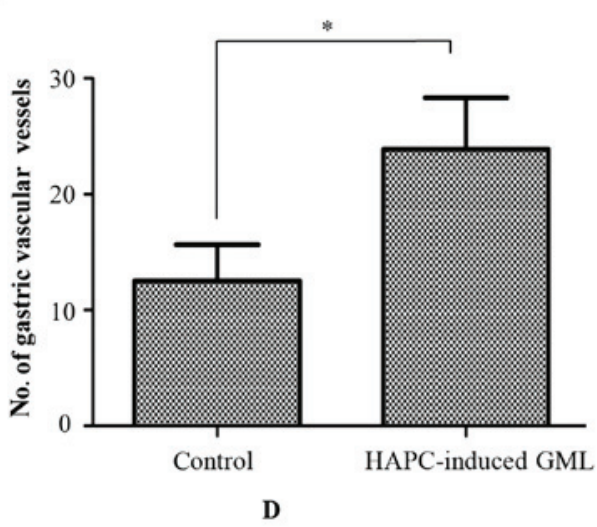

GML

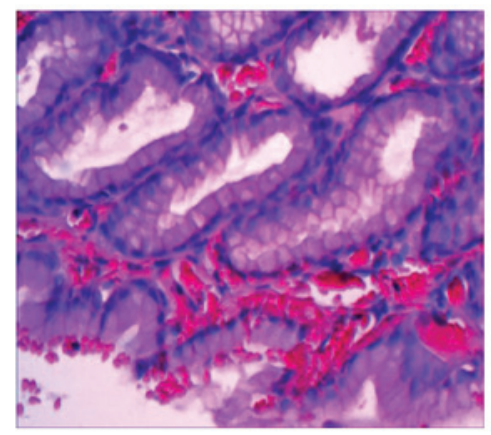

C

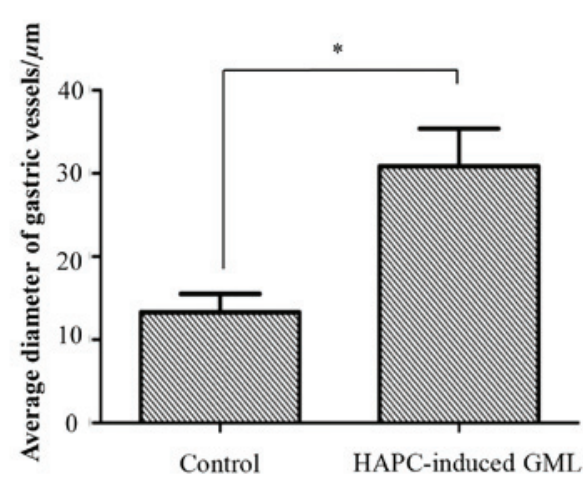

$*$

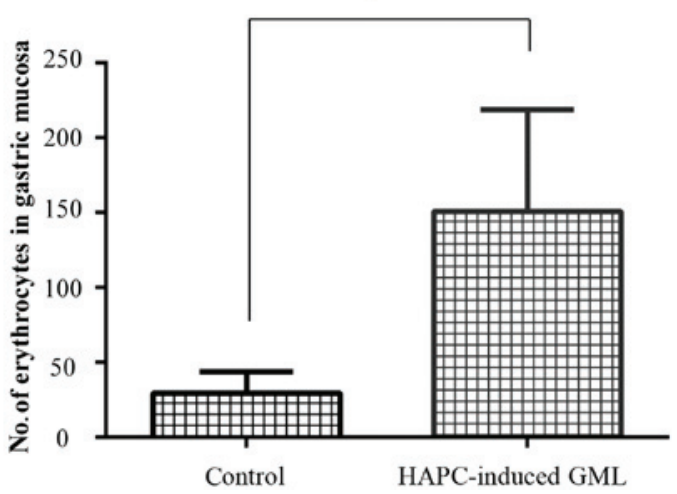

Figure 2. Histopathological changes in gastric mucosa sections. (A) Hematoxylin-eosin staining of gastric mucosa in the antrum region of the HAPC-induced GML group and the control group (magnification, x400). (B) Number of gastric vascular vessels in the antrum region of the HAPC-induced GML group and the control group. (C) Average diameter of gastric vessels in the antrum region. (D) Number of erythrocytes in the gastric mucosa of each group. Values are expressed as the mean \pm standard deviation $(n=3)$. "P<0.05. HAPC, high-altitude polycythemia; GMC, gastric mucosal lesions.

analysis was used to assess cell apoptosis in the gastric mucosal cells. The results showed that the amount of apoptotic gastric cells from the stomach of HAPC-induced GML patients was higher than that in healthy controls (Fig. 3A). To further validate the results above, the expression of apoptotic genes was assessed by RT-qPCR. The results showed that the expression caspase-3 and caspase-7 was significantly higher in the gastric tissues of patients with HAPC-induced GML than that in the healthy controls; furthermore, Bax was slightly but not significantly increased (Fig. 3B). These results suggested that cell apoptosis occurred in the gastric tissues of patients with HAPC-induced GML.

Mechanism of cell apoptosis in HAPC-induced GML. To explore the mechanism of cell apoptosis in HAPC-induced GML, the expression of PI3K pathway-associated genes was assessed through RT-qPCR. The results showed that the expression of PTEN was significantly higher in the gastric tissues of HAPC-induced GML patients than that in the healthy controls, while the expression of PI3K was significantly lower. Akt was also decreased in the gastric tissues of HAPC-induced GML patients, but not significantly (Fig. 4A). In accordance with these results, western blotting and immunohistochemistry confirmed that the expression of PI3K was higher in the gastric tissues of HAPC-induced GML patients than that in the healthy controls (Fig. 4B). All of these results demonstrated that cell apoptosis in HAPC-induced GML may be associated with the PI3K pathway.

\section{Discussion}

On account of the high altitude-associated increase in RBC, Tibetans suffer from increased blood viscosity, 
A

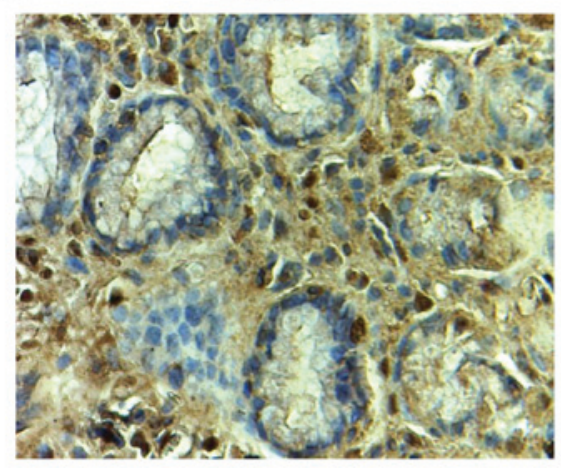

GML

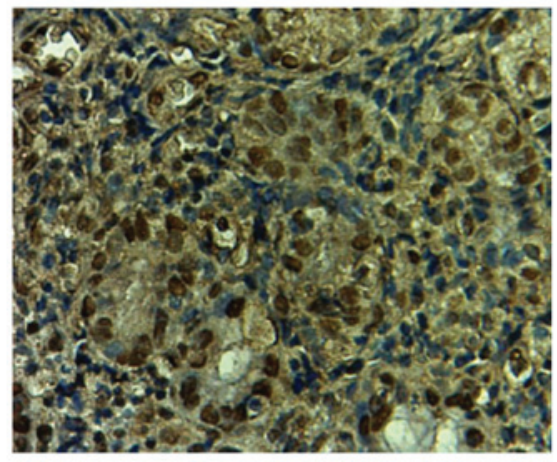

B

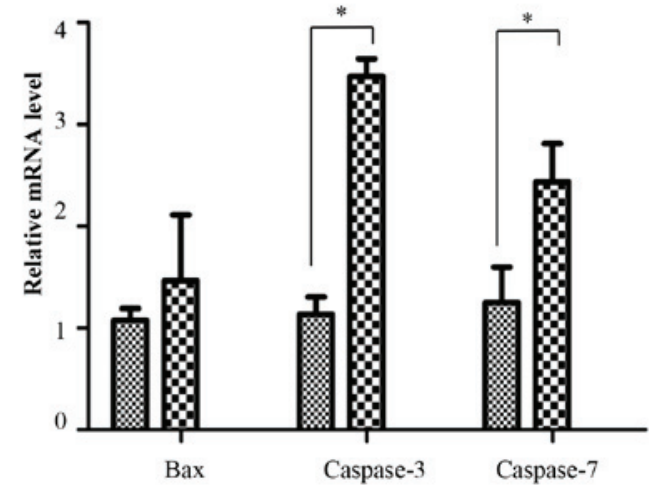

$\mathbf{W}$ HAPC-induced GML group

Figure 3. Cell apoptosis in HAPC-induced GML. (A) Terminal deoxynucleotidyl transferase-mediated deoxyuridine triphosphate nick-end labeling analysis was used to compare the amount of apoptotic gastric cells between HAPC-induced GML patients and healthy controls (magnification, $\mathrm{x} 400$ ). (B) Quantitative analysis of apoptotic molecules in gastric cells assessed by reverse-transcription quantitative polymerase chain reaction analysis. $\beta$-actin gene was used as an internal control. Values are expressed as the mean \pm standard deviation $(\mathrm{n}=3)$. ${ }^{*} \mathrm{P}<0.05$. HAPC, high-altitude polycythemia; GMC, gastric mucosal lesions; Bax, B-cell lymphoma 2-associated X protein.

A
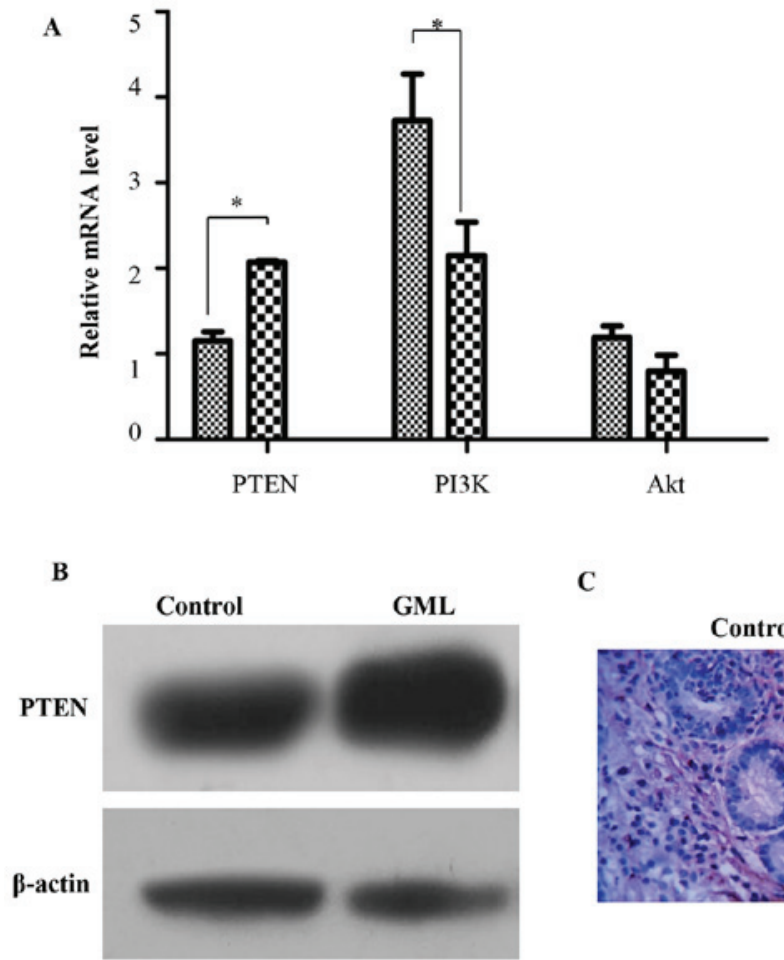

C

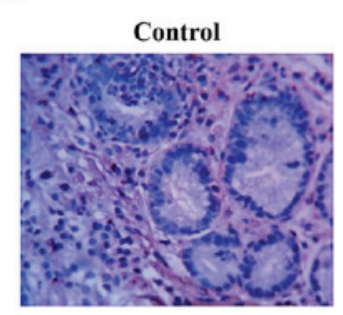

Control

W HAPC-induced GML group

Figure 4. Mechanism of GML induced by HAPC. (A) Quantitative analysis of PTEN, PI3K and Akt molecules in gastric cells assessed by reverse-transcription quantitative polymerase chain reaction analysis. $\beta$-actin gene was used as an internal control. Values are expressed as the mean \pm standard deviation ( $\mathrm{n}=3$ ). ${ }^{*} \mathrm{P}<0.05$. (B) Quantitative analysis of PTEN in gastric cells assessed by western blotting. (C) Quantitative analysis of PTEN in gastric cells assessed by immunohistochemistry (magnification, x400). HAPC, high-altitude polycythemia; GMC, gastric mucosal lesions; PTEN, phosphatase and tensin homolog; PI3K, phosphoinositide-3 kinase. 
microcirculation disturbances and systemic disorders. However, effective prevention and control measures for HAPC have yet to be utilized $(15,16)$. In addition, it has been reported that GML is the most frequent concomitant disease to HAPC. However, HAPC-induced GML and the underlying molecular mechanisms have remained to be fully elucidated. The present study assessed gastric tissues of patients with HAPC-induced GML and healthy controls through ultrastructural and histopathological analysis. Furthermore, TUNEL and RT-qPCR analysis were used to assess apoptosis in gastric mucosal cells. Finally, the expression of PI3K pathway-associated genes was determined by RT-qPCR in order to investigate the mechanism of cell apoptosis in HAPC-induced GML. The results indicated that a significant increase in the number of RBCs, gastric vessels and the diameter of gastric mucosal vessels in HAPC-induced GML patients compared with that in healthy controls. In addition, in HAPC-induced GML patients, RBCs had a higher distribution in gastric tissue not only at the vascular level but also in the tissue space. Furthermore, the number of vacuoles was increased in the gastric mucosal cells. A significant increase in apoptosis of the gastric mucosal cells was identified in HAPC-induced GML patients. Furthermore, the expression of PTEN was significantly higher in HAPC-induced GML patients than that in the healthy controls. These pathological changes suggested that apoptosis occurred in the stomach tissues of patients with HAPC-induced GML, which may be associated with the PI3K pathway.

Apoptosis is a major cell death pathway (17). Numerous studies have shown that apoptotic cell death of gastric mucosal cells has an important role in GML (18-20). In line with this, the present study found that the expression of Bax, caspase-3 and caspase-7 was higher in the stomach tissues of HAPC-induced GML patients than that in the healthy controls, suggesting that cell apoptosis also takes an active part in HAPC-induced GML.

The PI3K pathway participates in various cellular processes, including proliferation, transformation, apoptosis, tumor growth and angiogenesis, through its downstream molecule Akt $\backslash$ mammalian target of rapamycin (mTOR) (21-23). The tumor suppressor PTEN is a plasma-membrane lipid phosphatase, which antagonizes the function of PI3K by dephosphorylating phosphatidylinositol 3,4,5-trisphosphate back to phosphatidylinositol 4,5-bisphosphate to consequently inhibit the activation of Akt. PTEN therefore negatively regulates the PI3K/Akt/mTOR pathway to impact various cellular processes (24-26). In addition, the dysregulation of PTEN has been suggested to be closely associated with gastric carcinogenesis and pre-cancerous lesions by numerous studies (27-29). The present study detected that the expression of PTEN was significantly higher in the gastric tissues of HAPC-induced GML patients than that in the healthy controls through RT-qPCR, western blotting and immunohistochemistry. Furthermore, the expression of PI3K and Akt was significantly lower in the gastric mucosa of HAPC-induced GML patients than that in the healthy controls, which also indicated that the PI3K pathway was downregulated in HAPC-induced GML. All of these results illustrated that the upregulation of PTEN may induce gastric mucosal cell apoptosis through inhibiting the PI3K/Akt pathway in patients with HAPC-induced GML.

In conclusion, the present study showed that the microvessel density and the diameters of gastric mucosal vessels were significantly higher in gastric tissues of patients with HAPC-induced GML compared with those in healthy controls. The present study also provided a molecular mechanism of HAPC-induced GML, namely that upregulation of PTEN induces gastric mucosal cell apoptosis through inhibiting the PI3K/Akt pathway. However, further integrated analysis is required to provide more precise information regarding HAPC-induced GML. The results of the present study provide a basis for exploring treatment methods for gastric lesions caused by HAPC in the future.

\section{References}

1. León-Velarde F, Maggiorini M, Reeves JT, Aldashev A, Asmus I, Bernardi L, Ge RL, Hackett P, Kobayashi T, Moore LG, et al: Consensus statement on chronic and subacute high altitude diseases. High Alt Med Biol 6: 147-157, 2005.

2. Peyssonnaux C, Nizet V and Johnson RS: Role of the hypoxia inducible factors HIF in iron metabolism. Cell Cycle 7: 28-32, 2008.

3. Martin D and Windsor J: From mountain to bedside: Understanding the clinical relevance of human acclimatisation to high-altitude hypoxia. Postgrad Med J 84: 622-627, 2008.

4. Winslow RM: High-altitude polycythemia. In: High Altitude and Man. West JB, Lahiri S (eds). American Physiological Society, Bethesda, Md: pp163-172, 1984.

5. Laine L, Takeuchi K and Tarnawski A: Gastric mucosal defense and cytoprotection: Bench to bedside. Gastroenterology 135: 41-60, 2008.

6. Guth PH: Current concepts in gastric microcirculatory pathophysiology. Yale J Biol Med 65: 677-688, 1992.

7. Yoshida M, Wakabayashi G, Ishikawa H, Kawachi S, Tanabe M, Otani Y, Shimazu M, Kubota T and Kitajima M: Arteriovenous shunting blood flow is intravitally observed in the stomach after thermal injury in rats. Keio J Med 51: 193-200, 2002.

8. Kurata JH: Ulcer epidemiology: An overview and proposed research framework. Gastroenterology 96: 569-580, 1989.

9. Syam AF, Simadibrata M, Wanandi SI, Hernowo BS, Sadikin M and Rani AA: Gastric ulcers induced by systemic hypoxia. Acta Med Indones 43: 243-248, 2011.

10. Li K, Gesang L, Dan Z, Gusang L, Dawa C and Nie Y: Transcriptome reveals 1400 -fold upregulation of APOA4-APOC3 and 1100 -fold downregulation of GIF in the patients with polycythemia-induced gastric injury. PLoS One 10: e0140534, 2015.

11. Singh N, Deb R, Kashyap P, Bhatia V, Ahuja V and Sharma M: Body mass index and per capita income influence duodenal ulcer healing and $\mathrm{H}$. Pylori eradication whilst dietary factors play no part. Trop Gastroenterol 29: 26-31, 2008.

12. Fang JY, Liu WZ, Shi Y, Ge ZZ and Xiao SD: Consensus on chronic gastritis in China-second national consensus meeting on chronic gastritis (14-16 September 2006 Shanghai, China). J Dig Dis 8: 107-119, 2007.

13. Manninen P, Karvonen AL, Laukkarinen J, Aitola P, Huhtala $\mathrm{H}$ and Collin P: Colorectal cancer and cholangiocarcinoma in patients with primary sclerosing cholangitis and inflammatory bowel disease. Scand J Gastroenterol 50: 423-428, 2015.

14. Livak KJ and Schmittgen TD: Analysis of relative gene expression data using real-time quantitative PCR and the 2(-Delta Delta C(T)) Method. Methods 25: 402-408, 2001.

15. Jiang C, Chen J, Liu F, Luo Y, Xu G, Shen HY, Gao Y and Gao W: Chronic mountain sickness in Chinese Han males who migrated to the Qinghai-Tibetan plateau: Application and evaluation of diagnostic criteria for chronic mountain sickness. BMC Public Health 14: 701, 2014.

16. Fang J, Menon M, Kapelle W, Bogacheva O, Bogachev O, Houde E, Browne S, Sathyanarayana P and Wojchowski DM: EPO modulation of cell-cycle regulatory genes, and cell division, in primary bone marrow erythroblasts. Blood 110: 2361-2370, 2007.

17. Orrenius S, Zhivotovsky B and Nicotera P: Regulation of cell death: The calcium-apoptosis link. Nat Rev Mol Cell Biol 4: $552-565,2003$. 
18. Lam EK, Tai EK, Koo MW, Wong HP, Wu WK, Yu L, So WH, Woo PC and Cho CH: Enhancement of gastric mucosal integrity by Lactobacillus rhamnosus GG. Life Sci 80: 2128-2136, 2007.

19. Tsutsumi S, Tomisato W, Takano T, Rokutan K, Tsuchiya T and Mizushima T: Gastric irritant-induced apoptosis in guinea pig gastric mucosal cells in primary culture. Biochim Biophys Acta 1589: 168-180, 2002.

20. Brzozowski T, Konturek PC, Mierzwa M, Drozdowicz D, Bielanski W, Kwiecien S, Konturek SJ, Stachura J, Pawlik WW and Hahn EG: Effect of probiotics and triple eradication therapy on the cyclooxygenase (COX)-2 expression, apoptosis, and functional gastric mucosal impairment in helicobacter pylori-infected mongolian gerbils. Helicobacter 11: 10-20, 2006.

21. Engelman JA, Luo J and Cantley LC: The evolution of phosphatidylinositol 3-kinases as regulators of growth and metabolism. Nat Rev Genet 7: 606-619, 2006.

22. Janus A, Robak T and Smolewski P: The mammalian target of the rapamycin (mTOR) kinase pathway: Its role in tumourigenesis and targeted antitumour therapy. Cell Mol Biol Lett 10: 479-498, 2005.

23. Falasca M: PI3K/Akt signalling pathway specific inhibitors: A novel strategy to sensitize cancer cells to anti-cancer drugs. Curr Pharm Des 16: 1410-1416, 2010.

24. Vivanco I and Sawyers CL: The phosphatidylinositol 3-kinase-AKT pathway in human cancer. Nat Rev Cancer 2 : 489-501, 2002
25. Stambolic V, Suzuki A, De La Pompa JL, Brothers GM, Mirtsos C, Sasaki T, Ruland J, Penninger JM, Siderovski DP and Mak TW: Negative regulation of PKB/Akt-dependent cell survival by the tumor suppressor PTEN. Cell 95: 29-39, 1998

26. Martelli AM, Tazzari P, Evangelisti C, Chiarini F, Blalock WL, Billi AM, Manzoli L, McCubrey JA and Cocco L: Targeting the phosphatidylinositol 3-kinase/Akt/mammalian target of rapamycin module for acute myelogenous leukemia therapy: From bench to bedside. Curr Med Chem 14: 2009-2023, 2007.

27. Li YL, Tian Z, Wu DY, Fu BY and Xin Y: Loss of heterozygosity on 10q23.3 and mutation of tumor suppressor gene PTEN in gastric cancer and precancerous lesions. World J Gastroenterol 11: 285-288, 2005.

28. Yang Z, Yuan XG, Chen J, Luo SW, Luo ZJ and Lu NH: Reduced expression of PTEN and increased PTEN phosphorylation at residue Ser380 in gastric cancer tissues: A novel mechanism of PTEN inactivation. Clin Res Hepatol Gastroenterol 37: 72-79, 2013.

29. Li Y, He X, Zheng H, Wu D, Yang X, Xin Y and Fu B: Expression of PTEN encoding product in malignant lesions of gastric mucosa and its significance. Shijie Huaren Xiaohua Zazhi 11: 1294-1296, 2003 (In Chinese). 